\title{
Comparison of Different Saturation Pulse Types in a Series of Saturation Transfer Difference NMR Experiment Based on Cox-1 Enzyme and Ibuprofen Interaction
}

\author{
Nil E. Binbay ${ }^{1, *}$, Berrin Ziyadanoğulları \\ ${ }^{1}$ Department of Electronics, Vocational School, Dicle University, Turkey \\ ${ }^{2}$ Faculty of Science, Dicle University, Turkey
}

Copyright $\mathrm{C} 2017$ by authors, all rights reserved. Authors agree that this article remains permanently open access under the terms of the Creative Commons Attribution License 4.0 International License

\begin{abstract}
Here we report, comparison of two different saturation pulse types with different shapes, Gaussian pulse and Reburp pulse. Pulses are comparatively used in a series of STD-NMR experiments for determine which one is more effective for saturation and also saturation transfer for ibuprofen and COX1 mixture. Both pulses have been comparatively studied, and they are applied to identical samples in identical experimental conditions to see the difference. Also they are examined over different pulse parameters, like lengths and powers. Finally it is shown that, re-burp pulse gives more intensive STD-NMR signals than Gaussian pulse shape under same conditions and same samples. That means Reburp pulse shape causes more effective saturation than Gaussian pulse shape for COX enzyme and ibuprofen. Results are in accordance with literature.
\end{abstract}

Keywords STD-NMR, Cyclooxygenase Enzyme, Ibuprofen, Gaussian Pulse, Reburp Pulse

\section{Introduction}

Ibuprofen is a well-known and widely used member of NSAID's (Non-Steroidal Anti-Inflammatory Drugs). The main mechanism of ibuprofen is suppressing synthesis of Prostaglandin by inhibition COX (Cyclooxygenase) enzymes. [1] Ibuprofen inhibits COX enzymes by binding them. [1] Binding interaction between ibuprofen and COX enzymes is fast and reversible. [1, 2, 4] Therefore mixture of them is suitable for STD-NMR (Saturation Transfer Difference Nuclear Magnetic Resonance) experiments.

As it is discovered earlier, COX enzyme has two isoforms; COX-1 and COX-2.[2-6] It is known that, Ibuprofen binds both of $\mathrm{COX}$ isoforms, $\mathrm{COX}-1$ and $\mathrm{COX}-2$, almost equally [2-5]. There is no selectivity either any of COX enzymes for ibuprofen. So when it is used as an anti-inflammatory drug in humans, it inevitably inhibits both of COX enzyme isoforms simultaneously. However these two isoforms possess different functions in human body, such as; COX-1 mainly responsible from protection of gastric mucosa, while COX-2 mainly responsible from inflammation. [2-5] Therefore, like other typical NSAID's, ibuprofen causes serious gastrointestinal side effects, especially in long term use. In short terms, while it is treating inflammation by binding to COX-2, it causes major side effects by binding COX-1. [2,3] To overcome this problem, research groups have been trying to find some COX-2 selective novel anti-inflammatory drugs for a while, and it is still represents a major challenge for drug designers.

STD-NMR (Saturation Transfer Difference Nuclear Magnetic Resonance) is a highly sophisticated novel technique which focuses binding interaction of a macromolecule (enzyme, protein etc.) and a small ligand under specific conditions. [7] It is usually very hard to study with massive organic molecules like enzymes, proteins etc. in NMR. They typically include thousands of single atoms, therefore the NMR spectra of these macro molecules does not look like NMR spectra of small molecules. Typically there is no solid and individual big peaks like spectra of small molecules; instead there are many small and indistinguishable peaks, located a wide area in spectra. So it is very hard, even impossible to study such big molecules in NMR. But in many case, it turns to be very critical information whether a small Ligand binds to a macro molecule or not, especially novel drug design studies. Typically it is wondered that, for example a novel drug candidate ligand, whether interact (bind) with big molecule target (enzyme or protein) or not. At this point STD-NMR 
technique emerges as a novel technique which has unique benefits to study with target big molecules and novel small ligands. It is highly useful for such drug design studies. It could be being revealed that with STD -NMR, which compound binds to macro molecule, and therefore shows more activity as a novel drug candidate. If the ligand binds, technique also can give some information about which proton groups of Ligand play more effective role in binding process. This information known as epitope mapping. [7]

STD-NMR based on selectively saturation of macro molecule and then observing only ligand's peaks to determine whether it binds or not. Therefore it does not necessary to be deal with complex NMR spectra of macro molecule. This fact is one of the major benefits of STD-NMR.STD-NMR technique also has some unique advantages over the others, such as observing enzymes and ligands in a solution and near-natural conditions, or requiring as little enzyme as nano-molar levels etc. Usage of this technique increases rapidly due to these advantages. [7, 8]

In STD-NMR, determining and calibrating of saturation pulse parameters (shape, duration, power etc.) for each experiment is one of the very important steps in experimental process and could cause dramatic effect on success of the experiment. For example using not optimised saturation pulse power could easily cause false results. If saturation pulse power being applied higher than necessary, you may excite ligands peaks so you get false positive STD signals. On the other hand, if you set the saturation pulse power very low then you may could not saturate enzymes peaks effectively, and you get false negatives. So optimising saturation pulse parameters is necessary for STD-NMR experiments. And once you could not see the macro molecule's peaks directly, it is very hard to be sure if macro molecule is saturated or not. Every effort which contributes to find correct set of these parameters, is also contributes success of experiments and indirectly contributes to novel drug design processes, whose uses this technique.

The main aim of this work is to determine which pulse type is more effective in such a STD experiment including COX enzyme and ibuprofen. And also to analyse effects of pulse parameters and to find optimum parameter set to get improved STD-NMR signals.

\section{Experimental}

The COX-1 isoform from ram seminal vesicles and ibuprofen were purchased from Sigma-Aldrich. Ibuprofen was supplied as dry powder and $20 \mathrm{mM}$ ibuprofen stock solutions were prepared with solving ibuprofen in DMSO-d6. COX-1 isoform from ram seminal vesicles were supplied in $80 \mathrm{mM}$ pH 8.0 Tris- $\mathrm{HCl}, 0.1 \%$ Polysorbate 20 , and $300 \mu \mathrm{M}$ diethyldithiocarbamate (DDC), and used as such. Then $400 \mu \mathrm{L}$ of ibuprofen stock solution, COX-1 Enzyme solution and 100 $\mu \mathrm{L}$ of Tris- $\mathrm{HCl}(\mathrm{pH} 8.0)$ as buffer were mixed in a $5 \mathrm{~mm}$ NMR tube. And then STD-NMR experiments are carried out in BRUKER AVANCE III $400 \mathrm{MHz}$ spectrometer. Off resonance and on resonance frequencies are selected as 36 $\mathrm{kHz}$ and $-200 \mathrm{~Hz}$ (90 ppm, -0,5 ppm). "Stddiffgp19.2” pulse program was used from Bruker's pulse program library for all experiments. All spectra were recorded with 1024 scans.

\section{Results}

\subsection{Gaussian Saturation Pulse Shape with Different Pulse Powers}

Gaussian shaped saturation pulses were applied as $50 \mathrm{~ms}$ pulse trains for 4 seconds along the saturation time to the ibuprofen and COX1 mixture. Off resonance and on resonance frequencies are selected as $36 \mathrm{kHz}$ and $-200 \mathrm{~Hz}$ (90 ppm, -0,5 ppm). "Stddiffgp19.2" pulse program routine was used from Bruker's pulse program library for STD-NMR experiment. Pulse power was varied from $30 \mathrm{~dB}$ to $80 \mathrm{~dB}$. Spectra were recorded with 1024 scans (with 64 dummy scans). Results are shown in figure-1 and figure 2. 


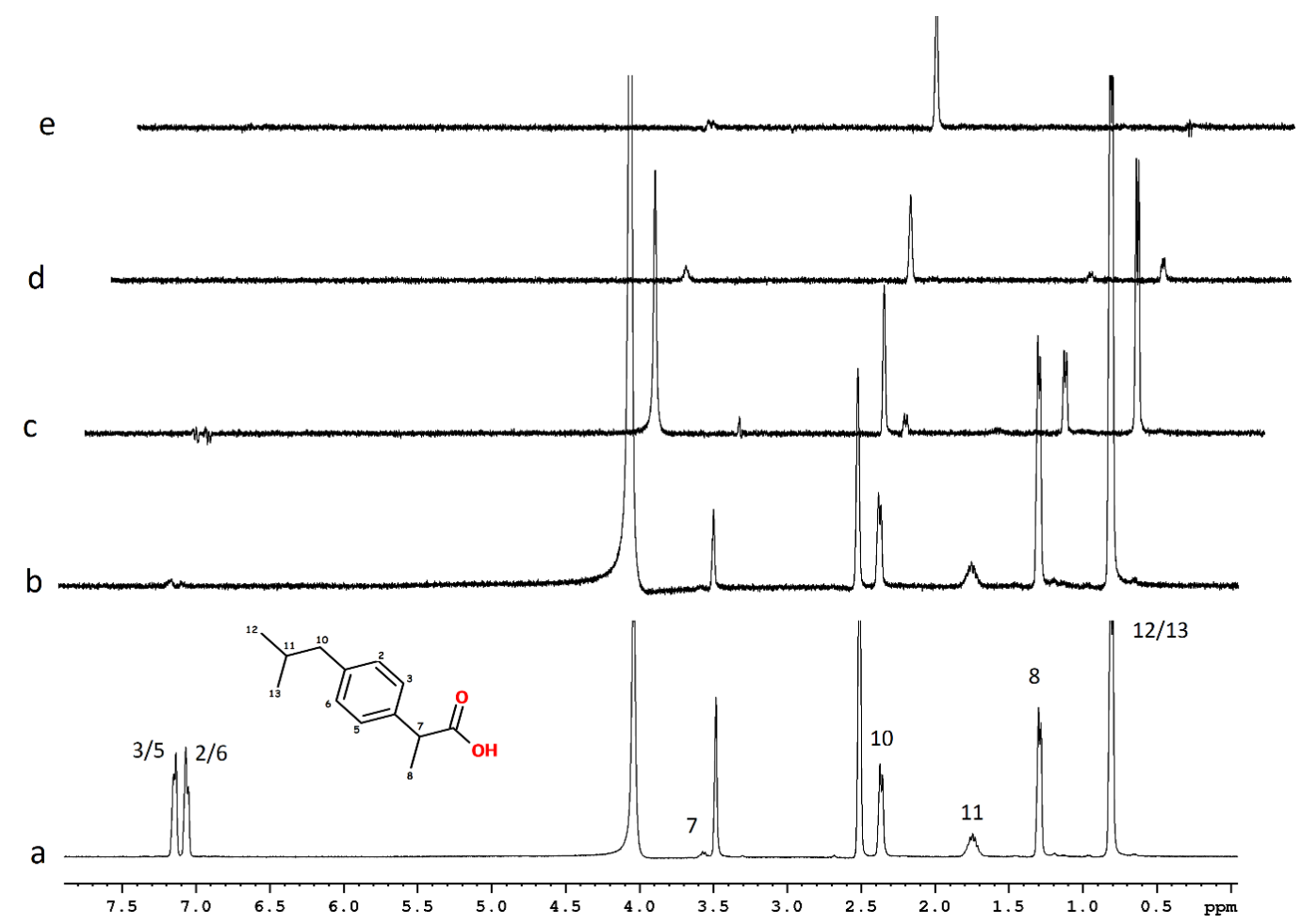

Figure 1. (a)1D proton NMR spectrum of ibuprofen; (b) STD-NMR spectrum of ibuprofen and COX1 mixture with Gaussian pulse shape, with $30 \mathrm{~dB}$ pulse power and $50 \mathrm{~ms}$ pulse length; (c) STD-NMR spectrum of ibuprofen and COX1 mixture with Gaussian pulse shape, with $40 \mathrm{~dB}$ pulse power and $50 \mathrm{~ms}$ pulse length; (d) STD-NMR spectrum of ibuprofen and COX1 mixture with Gaussian pulse shape, with $50 \mathrm{~dB}$ pulse power and $50 \mathrm{~ms}$ pulse length; (e) STD-NMR spectrum of ibuprofen and COX1 mixture with Gaussian pulse shape, with $80 \mathrm{~dB}$ pulse power and $50 \mathrm{~ms}$ pulse length.

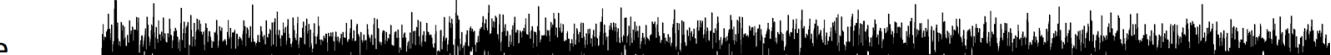

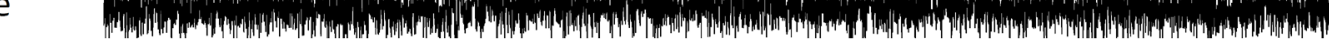

d

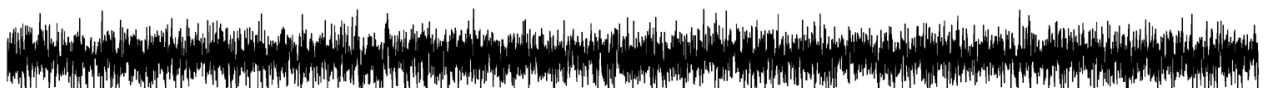

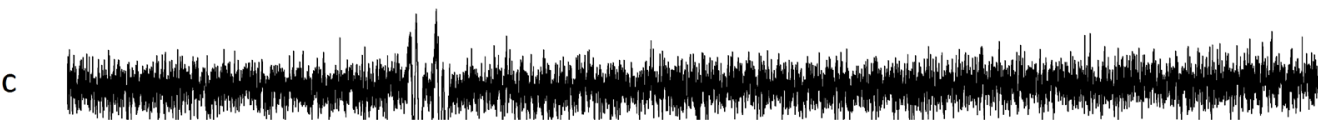

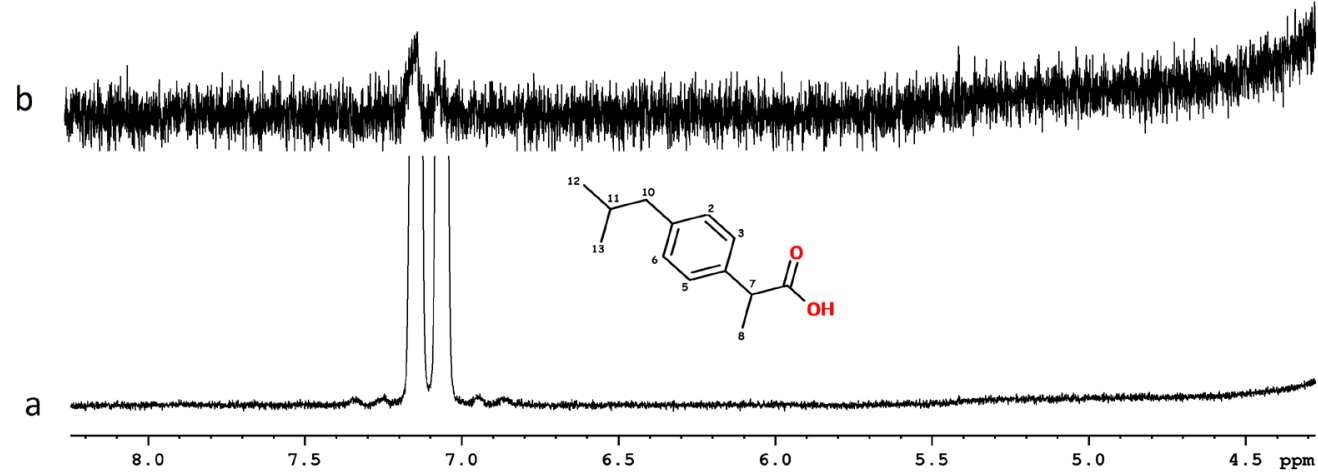

Figure 2. (a)1D proton NMR spectrum of ibuprofen (aromatic region of spectrum, low field); (b) STD-NMR spectrum of ibuprofen and COX1 mixture with Gaussian pulse shape, with $30 \mathrm{~dB}$ pulse power and $50 \mathrm{~ms}$ pulse length (aromatic region of spectrum, low field); (c) STD-NMR spectrum of ibuprofen and COX1 mixture with Gaussian pulse shape, with $40 \mathrm{~dB}$ pulse power and $50 \mathrm{~ms}$ pulse length (aromatic region of spectrum, low field); (d) STD-NMR spectrum of ibuprofen and COX1 mixture with Gaussian pulse shape, with $50 \mathrm{~dB}$ pulse power and $50 \mathrm{~ms}$ pulse length (aromatic region of spectrum, low field); (e) STD-NMR spectrum of ibuprofen and COX1 mixture with Gaussian pulse shape, with $80 \mathrm{~dB}$ pulse power and 50 ms pulse length (aromatic region of spectrum, low field). 


\subsection{Different Pulse Powers for Gaussian Pulse with 10 ms Pulse Duration}

Gaussian shaped saturation pulses were applied as $10 \mathrm{~ms}$ pulse trains for 4 seconds along the saturation time to the ibuprofen and COX1 mixture. Off resonance and on resonance frequencies are selected as $36 \mathrm{kHz}$ and $-200 \mathrm{~Hz}(90 \mathrm{ppm},-0,5$ ppm). "Stddiffgp 19.2" pulse program routine was used from Bruker's pulse program library for STD-NMR experiment. Pulse power was varied between $70 \mathrm{~dB}$ and $80 \mathrm{~dB}$. Spectra were recorded with 1024 scans (with 64 dummy scans). Results are shown in figure- 3 and figure- 4 and figure- 5 .
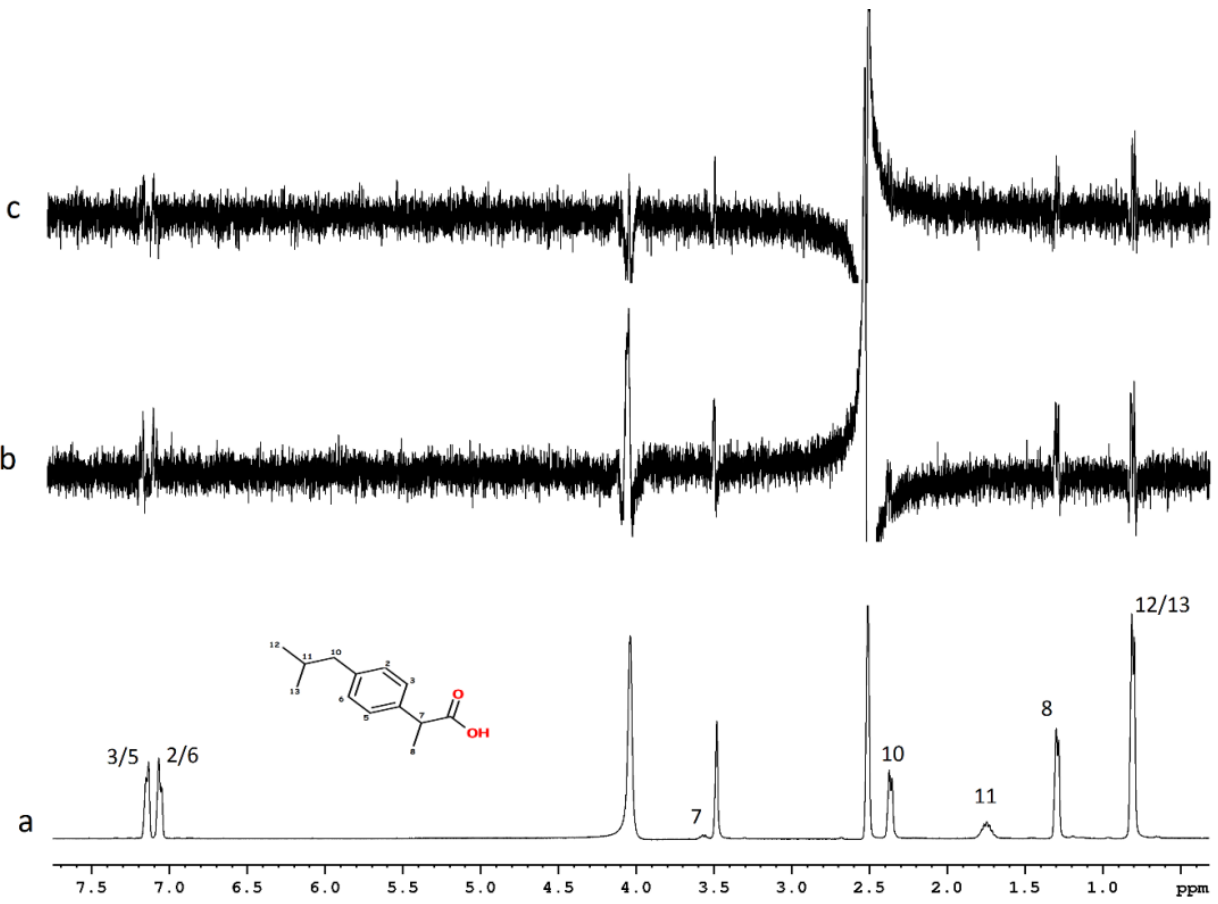

Figure 3. (a)1D proton NMR spectrum of ibuprofen; (b) STD-NMR spectrum of ibuprofen and COX1 mixture with Gaussian pulse shape, with $70 \mathrm{~dB}$ pulse power and $10 \mathrm{~ms}$ pulse length; (c) STD-NMR spectrum of ibuprofen and COX1 mixture with Gaussian pulse shape, with $80 \mathrm{~dB}$ pulse power and $10 \mathrm{~ms}$ pulse length.

C

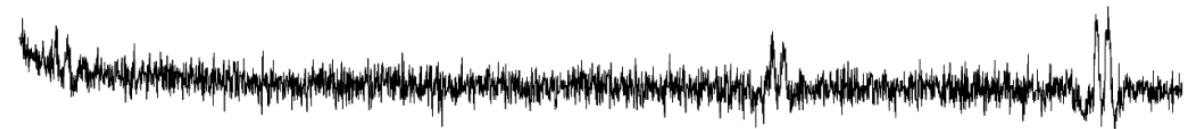

b
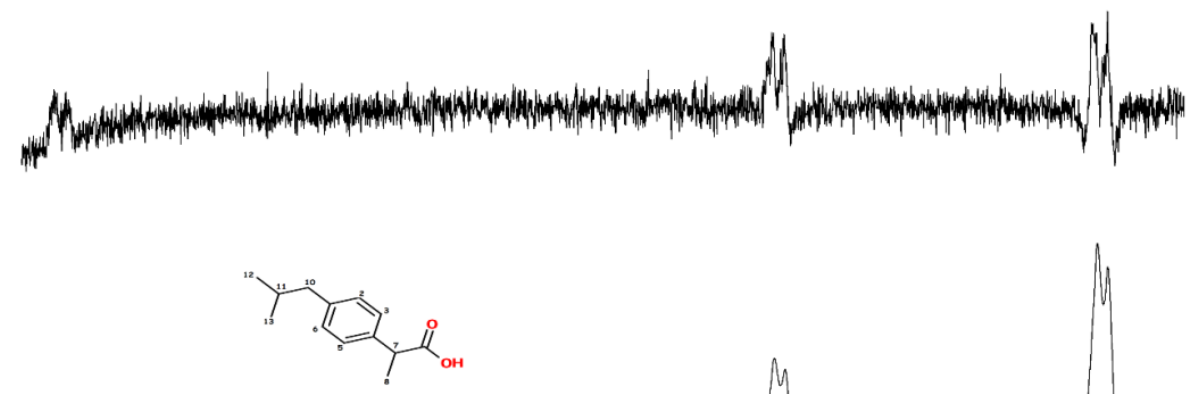

a

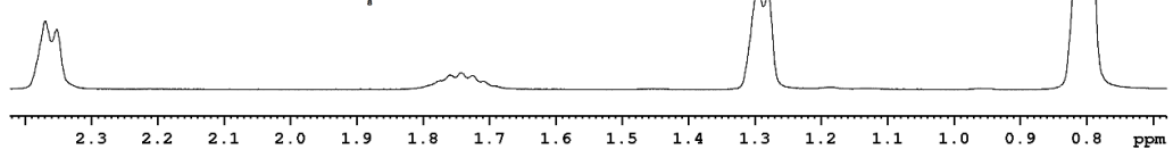

Figure 4. (a)1D proton NMR spectrum of ibuprofen (high field region of spectrum); (b) STD-NMR spectrum of ibuprofen and COX1 mixture with Gaussian pulse shape, with $70 \mathrm{~dB}$ pulse power and $10 \mathrm{~ms}$ pulse length (high field region of spectrum); (c) STD-NMR spectrum of ibuprofen and COX1 mixture with Gaussian pulse shape, with $80 \mathrm{~dB}$ pulse power and $10 \mathrm{~ms}$ pulse length (high field region of spectrum). 

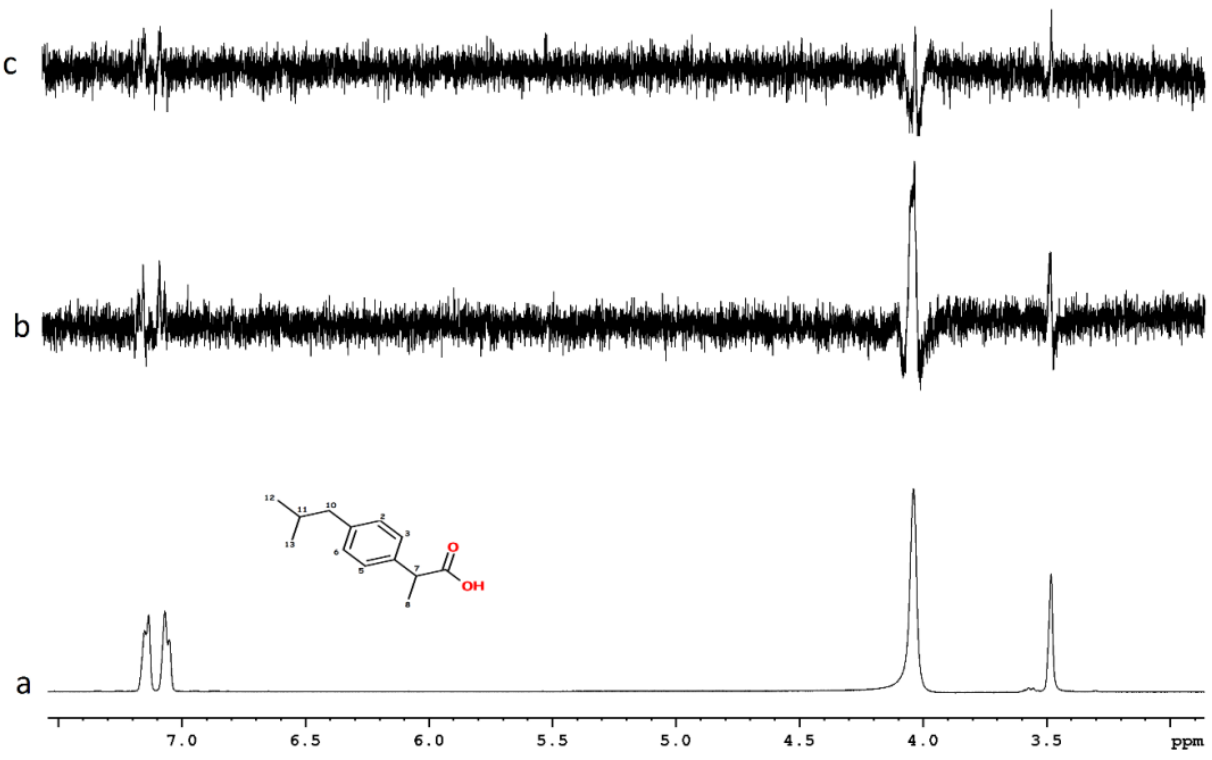

Figure 5. (a)1D proton NMR spectrum of ibuprofen (low field region of spectrum); (b) STD-NMR spectrum of ibuprofen and COX1 mixture with Gaussian pulse shape, with $70 \mathrm{~dB}$ pulse power and $10 \mathrm{~ms}$ pulse length (low field region of spectrum); (c) STD-NMR spectrum of ibuprofen and COX1 mixture with Gaussian pulse shape, with $80 \mathrm{~dB}$ pulse power and $10 \mathrm{~ms}$ pulse length (low field region of spectrum).

\subsection{Reburp Saturation Pulse Shape with Different Pulse Powers}

Reburp shaped saturation pulses were applied as 10 ms pulse trains for 4 seconds along the saturation time. Pulse power applied as $70 \mathrm{~dB}$ and $80 \mathrm{~dB}$. Spectra were recorded with 1024 scans (with 64 dummy scans). Results are shown in figure-6, figure-7, and figure-8.

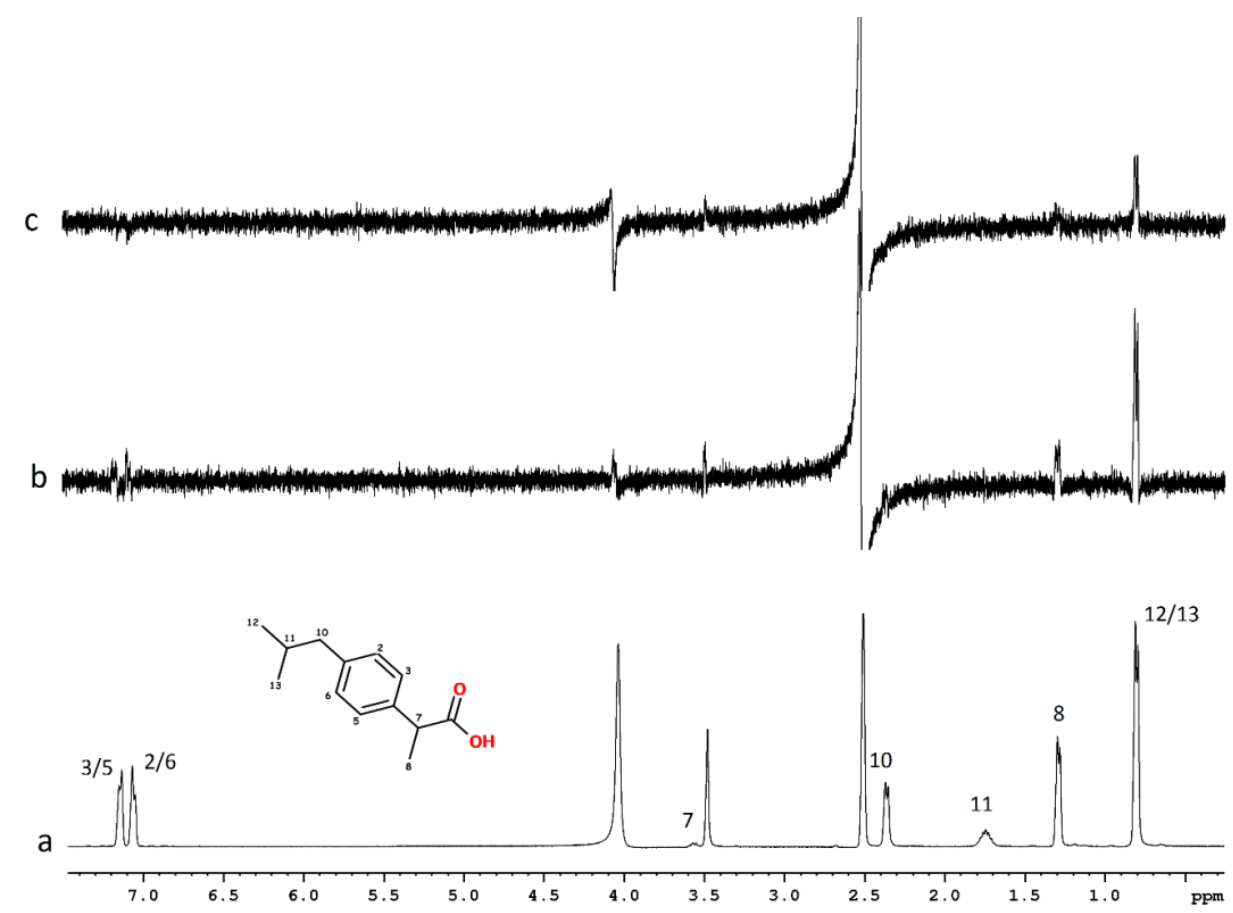

Figure 6. (a)1D proton NMR spectrum of ibuprofen; (b) STD-NMR spectrum of ibuprofen and COX1 mixture with Reburp pulse shape, with $70 \mathrm{~dB}$ pulse power and $10 \mathrm{~ms}$ pulse length; (c) STD-NMR spectrum of ibuprofen and COX1 mixture with Reburp pulse shape, with $80 \mathrm{~dB}$ pulse power and $10 \mathrm{~ms}$ pulse length. 
C

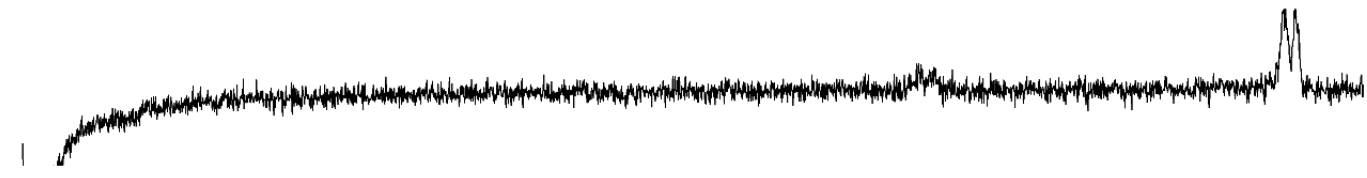

b

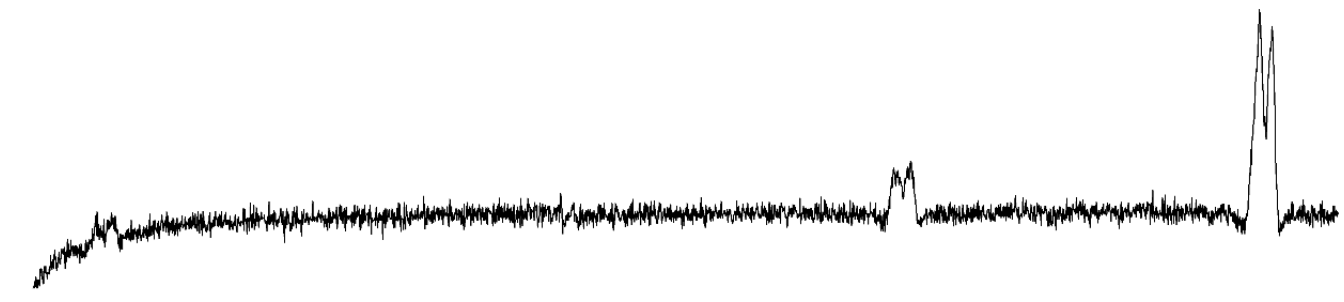

a

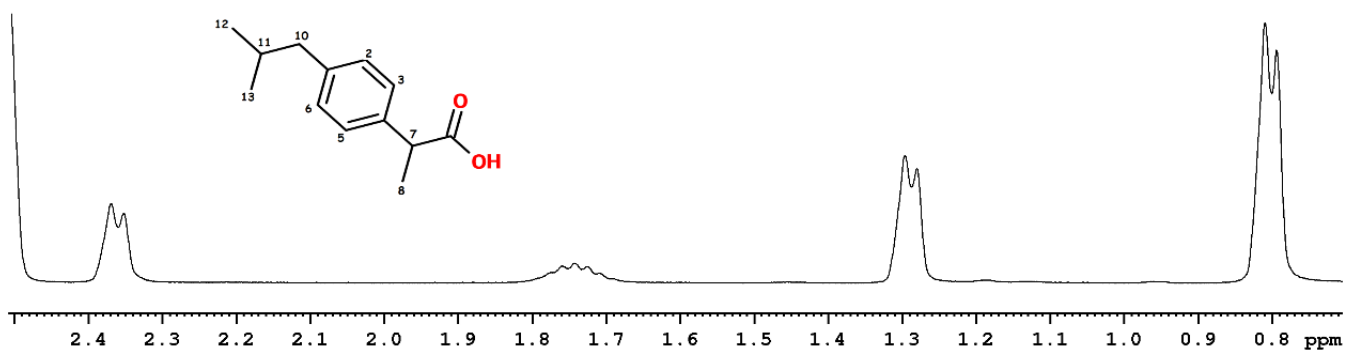

Figure 7. (a)1D proton NMR spectrum of ibuprofen (high field region of spectrum); (b) STD-NMR spectrum of ibuprofen and COX1 mixture with Reburp pulse shape, with $70 \mathrm{~dB}$ pulse power and $10 \mathrm{~ms}$ pulse length (high field region of spectrum); (c) STD-NMR spectrum of ibuprofen and COX1 mixture with Reburp pulse shape, with $80 \mathrm{~dB}$ pulse power and $10 \mathrm{~ms}$ pulse length (high field region of spectrum).
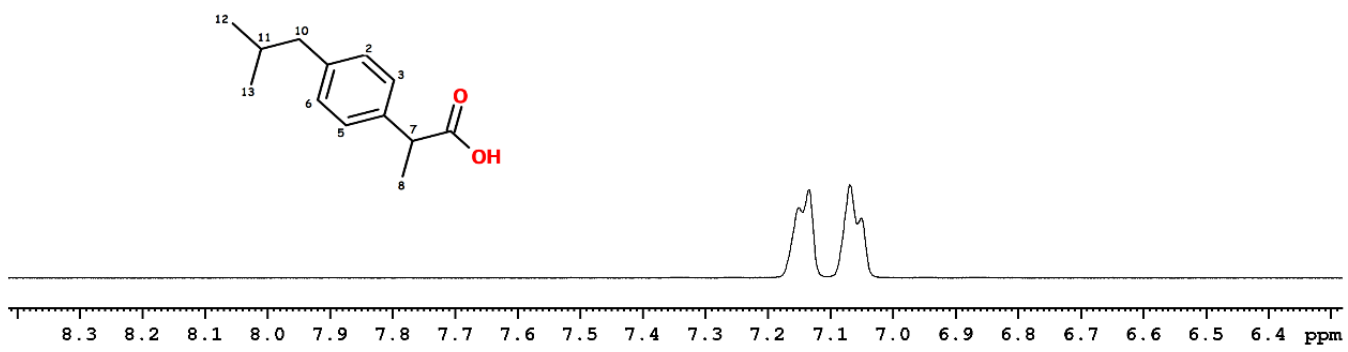

Figure 8. (a)1D proton NMR spectrum of ibuprofen (low field region of spectrum); (b) STD-NMR spectrum of ibuprofen and COX1 mixture with Gaussian pulse shape, with $70 \mathrm{~dB}$ pulse power and $10 \mathrm{~ms}$ pulse length (low field region of spectrum); (c) STD-NMR spectrum of ibuprofen and COX1 mixture with Gaussian pulse shape, with $80 \mathrm{~dB}$ pulse power and $10 \mathrm{~ms}$ pulse length (low field region of spectrum).

\subsection{Comparison of Gaussian and Reburp Pulses at $70 \mathrm{db}$ with $10 \mathrm{~ms}$ Pulse Duration}

Reburp and gaussian saturation pulses were both applied as $10 \mathrm{~ms}$ pulse trains for 4 seconds along the saturation time and same pulse power applied as $70 \mathrm{~dB}$ to compare each other. Spectra were recorded with 1024 scans (with 64 dummy scans). Results are shown in figure-9, figure-10 and figure- 11 . 
C

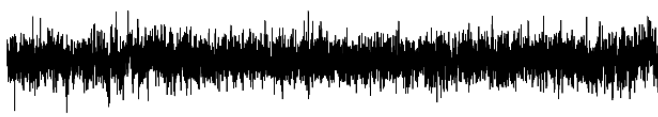

b
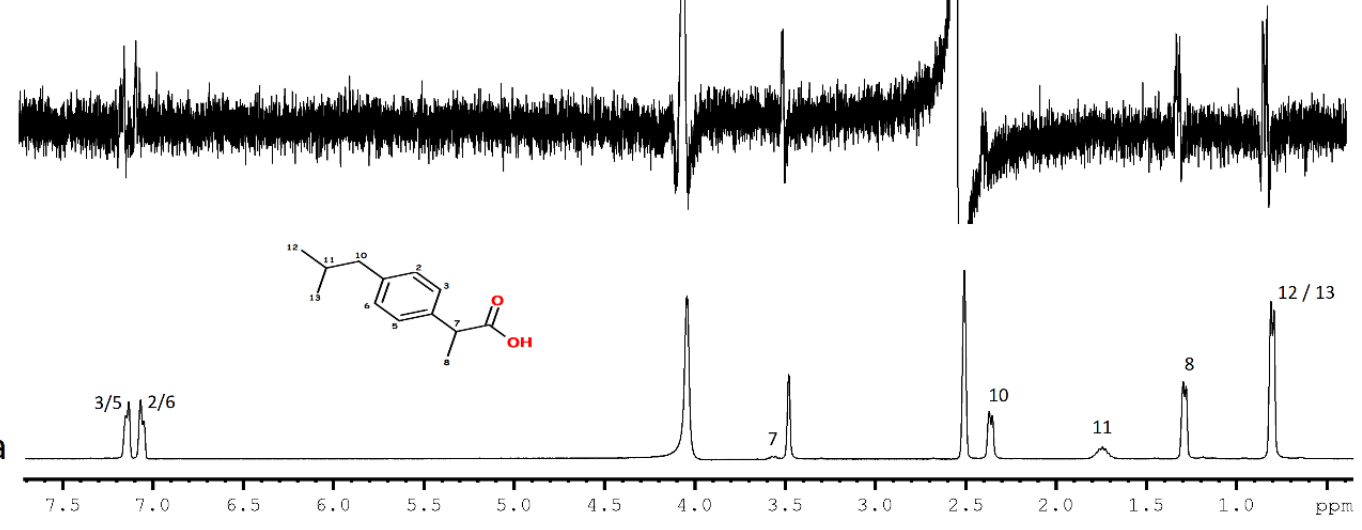

a

Figure 9. (a)1D proton NMR spectrum of ibuprofen; (b) STD-NMR spectrum of ibuprofen and COX1 mixture with Reburp pulse shape, with $70 \mathrm{~dB}$ pulse power and $10 \mathrm{~ms}$ pulse length; (c) STD-NMR spectrum of ibuprofen and COX1 mixture with Gaussian pulse shape, with $70 \mathrm{~dB}$ pulse power and $10 \mathrm{~ms}$ pulse length.

C
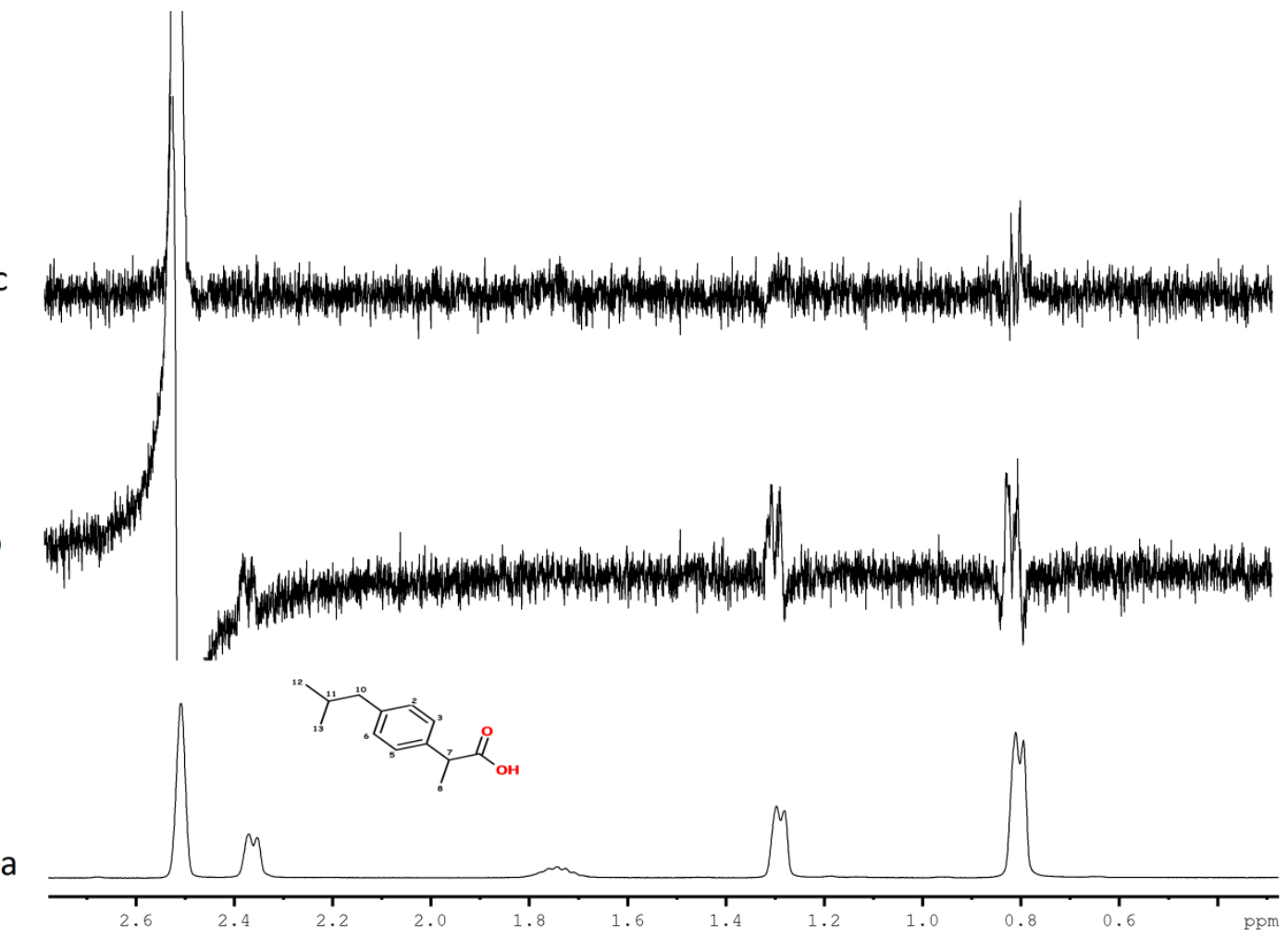

Figure 10. (a)1D proton NMR spectrum of ibuprofen (high field region of spectrum); (b) STD-NMR spectrum of ibuprofen and COX1 mixture with Reburp pulse shape, with $70 \mathrm{~dB}$ pulse power and $10 \mathrm{~ms}$ pulse length (high field region of spectrum); (c) STD-NMR spectrum of ibuprofen and COX1 mixture with Gaussian pulse shape, with $70 \mathrm{~dB}$ pulse power and $10 \mathrm{~ms}$ pulse length (high field region of spectrum). 
C

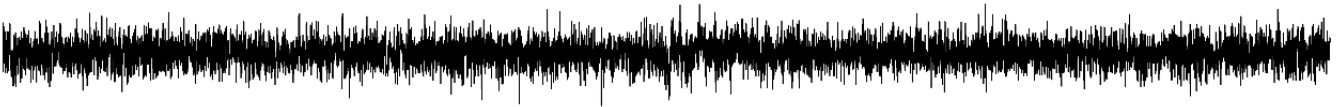

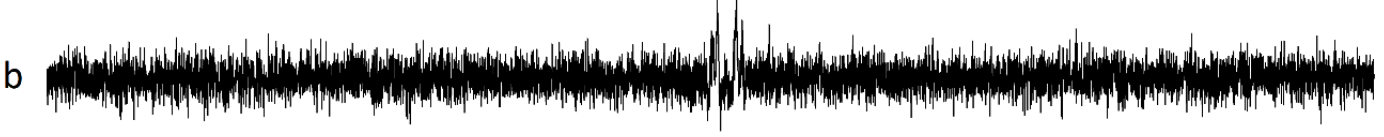

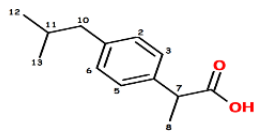

a

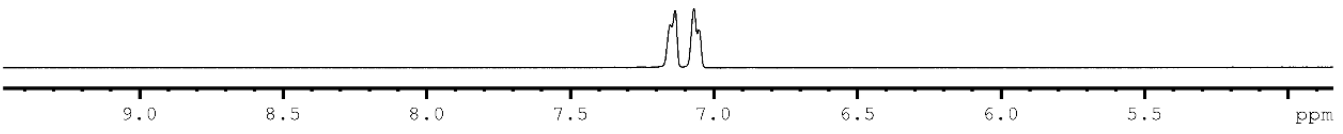

Figure 11. (a)1D proton NMR spectrum of ibuprofen (low field region of spectrum); (b) STD-NMR spectrum of ibuprofen and COX1 mixture with Reburp pulse shape, with $70 \mathrm{~dB}$ pulse power and $10 \mathrm{~ms}$ pulse length (low field region of spectrum); (c) STD-NMR spectrum of ibuprofen and COX1 mixture with Gaussian pulse shape, with $70 \mathrm{~dB}$ pulse power and $10 \mathrm{~ms}$ pulse length (low field region of spectrum).

\section{Discussion}

There are five spectra can be seen in figure-1: (a)1D proton NMR spectrum of ibuprofen, which is used as reference spectrum for observing STD spectra; (b) STD-NMR spectrum of ibuprofen and COX1 mixture with Gaussian saturation pulse shape applied, with $30 \mathrm{~dB}$ pulse power and 50 ms pulse length; (c) STD-NMR spectrum of ibuprofen and COX1 mixture with Gaussian saturation pulse shape applied, with $40 \mathrm{~dB}$ pulse power and $50 \mathrm{~ms}$ pulse length; (d) STD-NMR spectrum of ibuprofen and COX1 mixture with Gaussian saturation pulse shape applied, with $50 \mathrm{~dB}$ pulse power and $50 \mathrm{~ms}$ pulse length; (e) STD-NMR spectrum of ibuprofen and COX1 mixture with Gaussian pulse shape, with $80 \mathrm{~dB}$ pulse power and $50 \mathrm{~ms}$ pulse length.

It is clearly seen in the spectra, while saturation pulse power decreasing from $30 \mathrm{~dB}$ to $80 \mathrm{~dB}$, ((b) represents highest power at $30 \mathrm{~dB}$ and (e) represents lowest power at 80 $\mathrm{dB}$, (c) and (d) are $40 \mathrm{~dB}$ and $50 \mathrm{~dB}$ respectively), STD signals decreasing in accordance also. At lowest power, 80 $\mathrm{dB}$, there is almost no saturation remains to be observed as STD signal. It is revealed that, the correct adjustment of saturation pulse power is an important step for such a STD-NMR experiment. If it is not adjusted correctly, then saturation signals probably being loosed at all, and the experiment probably gives false negative results.

Another noticeable fact in spectra is that, the solvent (DMSO-d6) peak at around 2,5 ppm, always being saturated, nearly independent about how powerful saturation pulse being applied, as it is expected.

There are five spectra can be seen in figure-2, and (a), (b), (c), (d) and (e) are same as figure-1, but focused only low field region (aromatic region) of spectra. As it is seen in spectra, aromatic ring protons were not saturated as much as the methyl protons at around $0,8 \mathrm{ppm}$; that means these proton groups do not play active role in binding process to COX enzyme as other groups do. But STD signal of aromatic protons still depends on saturation pulse power as others, decreases accordingly with decreasing power.

There are three spectra can be seen in figure-3: (a)1D proton NMR spectrum of ibuprofen, which is used as reference spectrum for observing STD spectra; (b) STD-NMR spectrum of ibuprofen and COX1 mixture with Gaussian saturation pulse shape applied, with $70 \mathrm{~dB}$ pulse power and $10 \mathrm{~ms}$ pulse length; (c) STD-NMR spectrum of ibuprofen and COX1 mixture with Gaussian saturation pulse shape applied, with $80 \mathrm{~dB}$ pulse power and $10 \mathrm{~ms}$ pulse length.

As it is seen in figure-3, while saturation pulse power decreases from $70 \mathrm{~dB}$ to $80 \mathrm{~dB}$, STD signals also decreases, just like figure-1 and figure-2. But when figure-3 and figure-1 compared, it is seen that, saturation pulse duration is also effective on resulted STD signals. Shorter saturation pulse duration ( =10 ms, in figure-3, spectra (b) and (c) ), saturates wider bandwidth in frequency area, so affects more of enzyme protons, therefore one get more intensive STD signals, than longer saturation pulse duration $(=50 \mathrm{~ms}$, in figure-1 and figure-2, spectra (d) and (e) ).

There are three spectra can be seen in figure-4, and (a), (b), and (c) are same as figure-3, but focused only high field region of spectra. It is seen that stronger saturation pulse power at $70 \mathrm{~dB}$ in (b) causes more intense STD signals, than weaker saturation pulse at $80 \mathrm{~dB}$ in (c).

There are three spectra can be seen in figure-5, and (a), (b), and (c) are same as figure-3, but focused only low field region of spectra. It is seen that stronger saturation pulse 
power at $70 \mathrm{~dB}$ in (b) causes more intense STD signals, than weaker saturation pulse at $80 \mathrm{~dB}$ in (c).

There are three spectra can be seen in figure-6: (a)1D proton NMR spectrum of ibuprofen, which is used as reference spectrum for observing STD spectra; (b) STD-NMR spectrum of ibuprofen and COX1 mixture with Reburp saturation pulse shape applied, with $70 \mathrm{~dB}$ pulse power and $10 \mathrm{~ms}$ pulse length; (c) STD-NMR spectrum of ibuprofen and COX1 mixture with Reburp saturation pulse shape applied, with $80 \mathrm{~dB}$ pulse power and $10 \mathrm{~ms}$ pulse length.

As it is seen in figure-6, dependence of STD signals on applied saturation pulse powers is still preserved in Reburp pulse shape as same as Gaussian pulse shape was; increasing saturation pulse power (from $80 \mathrm{~dB}$ (spectrum (c)); to $70 \mathrm{~dB}$ (spectrum (b)) gives more intense STD signals, just as expected. But if figure- 6 and figure- 3 compared, it is seen that, Reburp pulse shape, with same pulse length, gives more intense STD signals, than Gaussian pulse shape does.

There are three spectra can be seen in figure-7, and (a), (b), and (c) are same as figure-6, but focused only high field region of spectra. It is seen that stronger saturation pulse power at $70 \mathrm{~dB}$ in (b) gives more intense STD signals, than weaker saturation pulse at $80 \mathrm{~dB}$ in (c).

There are three spectra can be seen in figure- 8 , and (a), (b), and (c) are same as figure-6, but focused only low field region of spectra. It is seen that stronger saturation pulse power at $70 \mathrm{~dB}$ in (b) gives more intense STD signals, than weaker saturation pulse at $80 \mathrm{~dB}$ in (c).

There are three spectra can be seen in figure-9: (a) 1D proton NMR spectrum of ibuprofen, which is used as reference spectrum for observing STD spectra; (b) STD-NMR spectrum of ibuprofen and COX1 mixture with Reburp saturation pulse shape applied, with $70 \mathrm{~dB}$ pulse power and $10 \mathrm{~ms}$ pulse length; (c) STD-NMR spectrum of ibuprofen and COX1 mixture with Gaussian saturation pulse shape applied, with $70 \mathrm{~dB}$ pulse power and $10 \mathrm{~ms}$ pulse length. It is clearly seen in figure- 9 that, Reburp shaped saturation pulse (spectrum (b)) gives more intense STD signals, than Gaussian shaped saturation pulses with exactly same conditions for COX1 enzyme and ibuprofen mixture, in such a STD-NMR experiment.

There are three spectra can be seen in figure-10, and (a), (b), and (c) are same as figure-9, but focused only low field region of spectra. It is seen that, STD signal of ibuprofen's peak-10 at around 2,37 ppm can only be seen by Reburp saturation pulse being applied (spectrum (b)), and it is not visible with Gaussian saturation pulse (spectrum (c)).

There are three spectra can be seen in figure-11, and (a), (b), and (c) are same as figure-9, but focused only high field region of spectra. It is seen that, STD signal of ibuprofen's aromatic peaks at around 7,2 ppm can only be seen by Reburp saturation pulse being applied (spectrum (b)), and it is not visible with Gaussian saturation pulse (spectrum (c)).

Although the exact interaction mechanism between ibuprofen and the COX enzymes does not being understood fully and still represents a major subject of some researches, it is commonly agreed that, ibuprofen binds to COX enzymes. $[9,10]$ Every spectrum in this work supports this fact, since STD signals, by definition, can only be being obtained if ligand (ibuprofen) binds to macromolecule (COX1 enzyme); otherwise there would be no STD signal at all, to be being observed. It is obviously seen that there are STD signals almost in every spectra. Existence of these signals are the experimental proof of the fact that ibuprofen binds to COX1.

\section{Conclusions}

Here we report a comparative study on differently shaped saturation pulses in a series of STD-NMR experiments, based on COX enzyme and ibuprofen binding interaction. These experiments are carried on with Gaussian and Reburp saturation pulse shapes applied to solution with different pulse powers, and different pulse lengths. Every single experiment takes approximately 10 hours of spectrometer time.

Finally it is concluded that, the Reburp pulse shape is gives more intense STD-NMR signals, therefore saturates more enzyme protons more effectively than Gaussian shaped saturation pulse in same experimental conditions, same pulse power and same pulse length for such a STD-NMR experiment includes COX enzyme and ibuprofen.

\section{Funding Sources}

This study is funded by DUBAP (Dicle University Scientific Research Projects Coordinatorship) under the Project No. 14-MYO-120.

\section{Acknowledgements}

Our special thanks go to DUBAP for funding this Project under the Project No: 14-MYO-120.

\section{REFERENCES}

[1] J. R. Vane., R. M. Botting. Anti-inflammatory drugs and their mechanism of action, Inflammation Res., Vol.47, Suppl.2, 78-87, 1998.

[2] L.A. Garcia Rodriguez, H. Jick. Risk of Upper Gastrointestinal Bleeding and Perforation Associated With Individual Non-Steroidal Anti-Inflammatory Drugs, Lancet, Vol.343, 769-772, 1994.

[3] M.M. Wolfe, D.R. Lichtenstein, G. Singh. Gastrointestinal Toxicity of Non-Steroidal Anti-Inflammatory Drugs, N. Engl. J. Med., Vol.340, 1888-1899, 1999.

[4] D.C. Brater. Clinical Aspects of Renal Prostaglandins and 
NSAID Therapy, Semin. Arthritis Rheum., Vol.17, 17-22, 1988.

[5] D. Kleinknecht, P. Landais, B. Goldfarb. Analgesic and Non-Steroidal Anti-Inflammatory Drug-Associated Acute Renal Failure: A Prospective Collaborative Study, Clin. Nephrol., Vol.25, 275-281, 1986.

[6] J.R. Vane, Y. S. Bakhle, M.R. Botting. Cyclooxygenases 1 and 2., Annu. Rev. Pharmacol Toxicol., Vol.38, 97-120, 1998.

[7] A.Viegas, J. Manso, F. L. Nobrega, E. J Cabrita. Saturation-Transfer Difference (STD) NMR: A Simple and Fast Method for Ligand Screening and Characterization of Protein Binding, J. Chem. Educ., Vol.88, 990-994, 2011.
[8] C. A. Lepre, J. M. Moore, J. W. Peng. Theory and Applications of NMR Based Screening in Pharmaceutical Research, Chem. Rev. Vol.104, 3641-3675, 2004.

[9] J. J. Prusakiewicz, K. C. Duggan, C. A. Rouzer, L. J. Marnett. Differential Sensitivity and Mechanism of Inhibition of COX-2 Oxygenation of Arachidonic Acid and 2-Arachidonoylglycerol by Ibuprofen and Mefenamic Acid, Biochemistry, Vol.48, No.31, 7353-7355, 2009.

[10] V. Limongelli, M. Bonomi, L. Marinelli, F. L. Gervasio, A. Cavalli, E. Novellino, M. Parrinello. Molecular Basis of Cyclooxygenase Enzymes (COXs) Selective Inhibition, PNAS, Vol.107, No.12, 5411-5416, 2010. 